\title{
Deep Speaker Embedding for Speaker-Targeted Automatic Speech Recognition
}

\author{
Guan-Lin Chao \\ Electrical and Computer Engineering \\ Carnegie Mellon University \\ 5000 Forbes Avenue, Pittsburgh, PA, USA 5000 Forbes Avenue, Pittsburgh, PA, USA \\ guanlinchao@cmu.edu
}

\author{
lan Lane \\ Electrical and Computer Engineering \\ Language Technologies Institute \\ Carnegie Mellon University \\ 5000 Forbes Avenue, Pittsburgh, PA, USA \\ lane@cmu.edu
}

\begin{abstract}
In this work, we investigate three types of deep speaker embedding as text-independent features for speaker-targeted speech recognition in cocktail party environments. The textindependent speaker embedding is extracted from the target speaker's existing speech segment ( $i$-vector and $x$-vector) or face image $(f$-vector $)$, which is concatenated with acoustic features of any new speech utterances as input features. Since the proposed model extracts the speaker embedding of the target speaker once and for all, it is computationally more efficient than many prior approaches which estimate the target speaker's characteristics on the fly. Empirical evaluation shows that using speaker embedding along with acoustic features improves Word Error Rate over the audio-only model, from $65.7 \%$ to $29.5 \%$. Among the three types of speaker embedding, $\mathrm{x}$-vector and f-vector show robustness against environment variations while $\mathrm{i}$-vector tends to overfit to the specific speaker and environment condition.
\end{abstract}

\section{CCS Concepts}

- Computing methodologies $\rightarrow$ Artificial intelligence $\rightarrow$ Natural language processing $\rightarrow$ Speech recognition

\section{Keywords}

speaker-targeted speech recognition; robust speaker embeddings; acoustic modeling.

\section{INTRODUCTION}

Robust speech recognition in cocktail party environments remains a challenging task in Automatic Speech Recognition (ASR). While current ASR systems have competitive performance in a low noise environment, their performance deteriorates when background noise or background speech is present. In this work, we focus on speaker-targeted speech recognition whose goal is to transcribe a target speaker from overlapping speech.

In many real-world applications, before a speaker starts talking, a speech recognizer (such as a social robot) may already have

Permission to make digital or hard copies of all or part of this work for personal or classroom use is granted without fee provided that copies are not made or distributed for profit or commercial advantage and that copies bear this notice and the full citation on the first page. Copyrights for components of this work owned by others than the author(s) must be honored. Abstracting with credit is permitted. To copy otherwise, or republish, to post on servers or to redistribute to lists, requires prior specific permission and/or a fee. Request permissions from Permissions@acm.org.

NLPIR 2019, June 28-30, 2019, Tokushima, Japan

(C) 2019 Copyright is held by the owner/author(s). Publication rights licensed to ACM.

ACM ISBN 978-1-4503-6279-5/19/06 ..\$15.00

DOI: https://doi.org/10.1145/3342827.3342847 access to the speaker's acoustic or visual data via one's mobile phones and social media, and could extract relevant speaker characteristics, ready to be used as additional features for speech recognition even before a speech signal is uttered by the target user. Moreover, the recognizer may know the speaker's identity by face recognition or various biometric identification methods and could use the identity to retrieve the speaker's information from the database of pre-extracted speaker embedding.

In this paper, we propose a pipeline of speaker-targeted acoustic model to recognize the speech of a target speaker from a mixture of speech signals $[2,5,10,14,24]$. The first part of the pipeline is speaker embedding extraction. The speaker embedding model will generate a fixed-dimensional vector, a speaker embedding, to represent the target speaker, based on a short segment of the speaker's speech or the speaker's face image. If the target speaker's identity is known, we can also use the identity as a cue to retrieve one's speaker embedding from the database. In the second part of the pipeline, we use the target speaker embedding as a "static" feature concatenated with the acoustic features of the current utterance as input to the acoustic model, as opposed to dynamically estimating the target speaker's characteristic along with the speech input stream, for the sake of transcribing efficiency (Section 3). We investigate three types of speaker embedding models which use a speech segment or face image as input to generate speaker embedding and compare their performance in different speaker and environment conditions (Section 4). Through empirical evaluation, we show that using speaker embedding reduces Word Error Rate (WER) significantly, from $65.7 \%$ to $29.5 \%$. Comparing the three types of speaker embedding, i-vectors tend to overfit to the exact speaker and environment condition and give the best performance when the speaker information environment matches that of speech recognition, while $\mathrm{x}$-vectors and f-vectors are more robust with respect to the environment variations, as they are trained to differentiate speakers (Section 5 and 6).

\section{RELATED WORK}

Speaker embedding models are widely used as the front-end of speaker verification and identification systems. The models are trained to map variable length speech utterances into a feature space. And the back-end classifiers use the projected embedding as input features to make decisions. Traditionally, Gaussian Mixture Model-Universal Background Models (GMM-UBMs) are used to extract i-vectors [4], which are useful speaker embedding to model speaker and channel variability at the same time. ivectors are generated by computing and optimizing sufficient statistics to fit the acoustic features of speech utterances. There have been many previous approaches to replacing the GMMUBM with Deep Neural Networks (DNNs) for speaker embedding extraction $[3,5,6,10-12,14,18,19,21-24]$. 
Speaker embedding is also useful for adapting acoustic models in speech recognition [1, 3, 5, 10, 12, 14, 21, 24]. [12] and [16] adapt DNN acoustic models to a target speaker by concatenating the input acoustic features with GMM-UBM-based and Hidden Markov Model-based i-vectors respectively. In [9], DNN acoustic models are adapted to noisy and reverberant acoustic environments for robust speech recognition, by concatenating acoustic features with environment embeddings, which are bottleneck features extracted from an environment recognition DNN. However, the aforementioned methods require the embedding estimation to be performed on the fly. In other words, after an utterance is received, the speech recognizer has to wait until the embedding is extracted from the current input signal, before transcribing starts.

\section{PIPELINE OF SPEAKER-TARGETED ACOUSTIC MODEL}

In this work, we focus on recognizing a target speaker's speech from overlapping speech signals of two speakers: the target and the background speaker. Here we describe our speaker-targeted acoustic modeling pipeline, as shown in Figure 1, to transcribe the speech of the target speaker, using the acoustic features of the overlapping as well as the target speaker's information.

In the first part of the pipeline, a speaker embedding model extracts a fixed-dimensional speaker embedding vector as representation of the target speaker, given the target speaker's acoustic or visual data. We assume that we have access to some acoustic or visual data of the target speaker via one's mobile phone or social media before the target speaker starts talking. The previously available data can be as simple as a segment of speech or a face image of the target speaker, in the same or different environment of the overlapping speech signal. Even before the overlapping speech signal comes in, we can use the previously available acoustic or visual data of the target speaker as input to our speaker embedding models to extract one's speaker characteristics, called speaker embedding. We can also pre-extract and store the speaker embedding in the database. And as long as the target speaker is identified (by face recognition or various biometric identification methods), our system can retrieve one's speaker embedding in advance.

In the second part of the pipeline, we supply an additional input feature: a static speaker embedding vector of the target speaker, concatenated with a window of acoustic frames (e.g. MFCC features) to extend the standard audio-only Time-Delay Neural Network (TDNN) acoustic model, which predicts the phoneme posterior probability. The reason for using a static embedding vector to represent the target speaker is to enhance transcribing efficiency, such that we don't have to dynamically estimate the target speaker's information along with the current input of overlapping speech.

\section{SPEAKER EMBEDDING MODELS}

We train and compare three types of text-independent speaker embedding models. i-vector and x-vector models are audio-based, taking a speech segment of the target speaker as input, and the fvector model is vision-based, taking a face image of the target speaker as input.

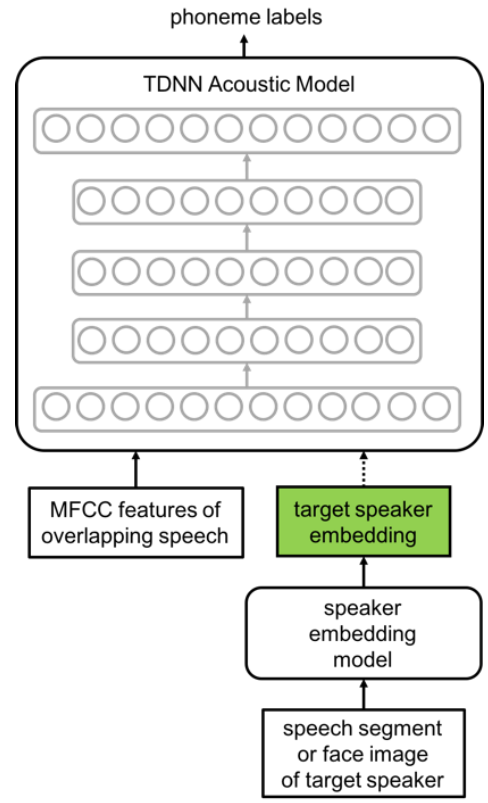

Figure 1: Acoustic models with Time Delay Neural Network (TDNN) architecture. The arrow connecting the target speaker embedding and the TDNN acoustic model is a dashed arrow. The audio-only model is illustrated without the dashed arrow. The speaker-targeted model is illustrated with the dashed arrow, where the target speaker embedding (green) can be extracted by a speaker embedding model given available target speaker data (a speech segment or face image), and supplied as additional input feature to the acoustic model.

\subsection{GMM-UBM i-vector Model}

The first speaker embedding model we consider is the conventional i-vector extractor. We use a 512-component diagonal GMM-UBM. Iteratively, we update the UBM components' means (factor loading submatrices), and collect sufficient statistics to estimate i-vectors, using the EM algorithm. Then we perform Linear Discriminant Analysis (LDA) to reduce the dimension of i-vectors to 128. i-vector extractor training is performed at frame-level MFCC features. In the inference phase, given an adaptation speech segment of the target speaker, we extract a frame-level i-vector every 10 frames, and the i-vector speaker embedding is extracted by taking the average of all the frame-level i-vectors across the entire segment.

\subsection{TDNN-based x-vector Model}

The second speaker embedding model is a speaker recognition model, with the same feed-forward deep neural network architecture, as in [19]. The input of the model is the MFCC features of all frames across a speech segment, and the output of the model is a speaker label. It is trained on multiclass cross entropy loss, to differentiate the 1228 speakers in the training set.

Below we describe the architecture of the model, as shown in Figure 2. The first four layers are time-delay and fully-connected layers which take a context of MFCC features as input to generate a frame-level representation. The middle statistical pooling layer aggregates all frame-level representations of the entire adaptation speech segment and concatenates the mean and standard deviation as statistical features. The last three layers are fully-connected layers and a softmax output layer for speaker classification. 
In the inference phase, we use the bottleneck features from the second to last hidden layer, projected through LDA to be a 128dimensional speaker embedding vector, which is called $\mathrm{x}$-vector.

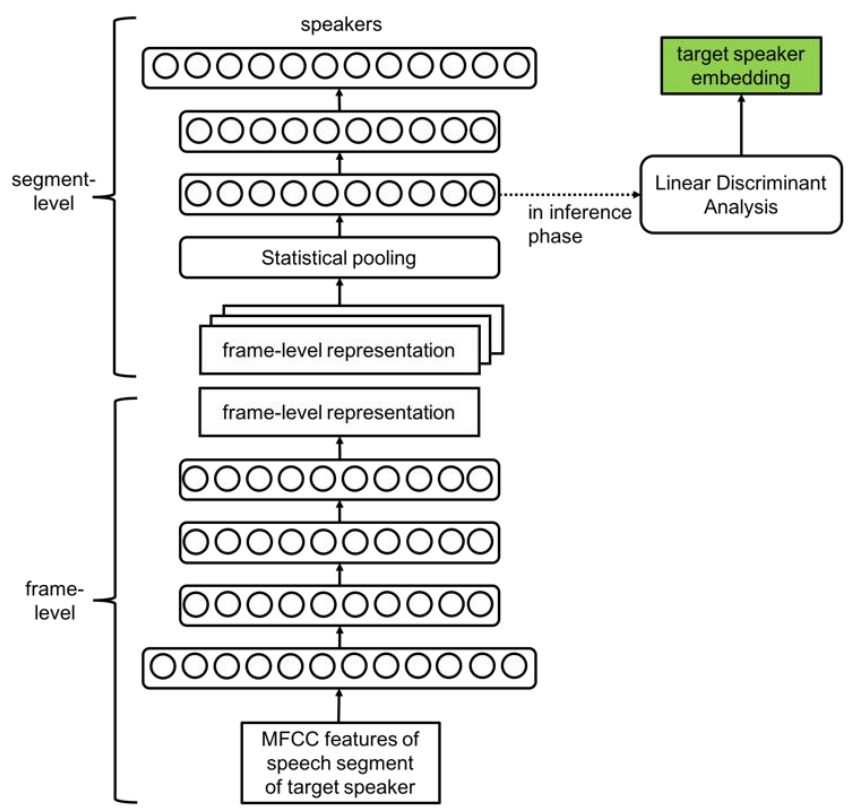

Figure 2: TDNN-based model extracts x-vectors from bottleneck features given the target speaker's speech segment as input. The TDNN model is pre-trained for speaker recognition.

\subsection{CNN-based f-vector Model}

The third speaker embedding model we consider is FaceNet, described in [17]. It is a deep Convolutional Neural Network (CNN) which takes face images as input and outputs a normalized 128-dimensional embedding. It is trained on triplet loss by supplying triplet training examples. A triplet consists of three face images: an anchor, a positive, and a negative image, where the anchor and positive images belong to the same speaker, and the negative image belongs to another. Triplet loss of a triplet training example is given by:

$$
\left[\left\|e^{a}-e^{p}\right\|_{2}^{2}-\left\|e^{a}-e^{n}\right\|_{2}^{2}+m\right]_{+}
$$

where $e^{a}, e^{p}, e^{n}$ denote the face embedding of the anchor, positive and negative face images, $m$ is the distance margin, and the operator $[x]_{+}=\max (x, 0)$. Training on triplet loss forces the network to project the face images that belong to the same speaker to have small Euclidean distances, and the projection of distinct speakers' face images to have large distances. We use a FaceNet model with the Inception ResNet v1 architecture, described in [20], which was pre-trained on the MS-Celeb-1M dataset [8], as shown in Figure 3. In the inference phase, we supply a face image of the target speaker as input to the FaceNet model and use the 128-dimensional vector output as speaker embedding. If multiple face images of the target speaker are provided, we use the average of all the 128-dimensional vector outputs as speaker embedding. We call this type of speaker embedding f-vector.



Figure 3: FaceNet extracts f-vectors from an input face image of the target speaker. FaceNet is a deep Convolutional Neural

Network of Inception-ResNet architecture pre-trained on triplet loss.

Table 1. The TED-LIUM corpus

\begin{tabular}{|l|c|c|c|}
\hline \multicolumn{1}{|c|}{ Characteristics } & Train & Dev & Test \\
\hline Number of videos & 1444 & 8 & 11 \\
\hline Number of utterances & 97876 & 507 & 1155 \\
\hline Duration of videos (hours) & 341.6 & 1.7 & 2.9 \\
\hline Number of speakers & 1228 & 8 & 11 \\
\hline
\end{tabular}

\section{EXPERIMENTS}

\subsection{Datasets}

For the dataset of single-speaker scenario, we directly use the real-world acoustic data from the TED-LIUM corpus release 2 [15]. We augment the dataset with the speaker face images. The speaker face images are obtained by applying face detection and tracking on the video frames of TED talks, which are downloaded from TED.com. Table 1 lists the statistics of the TED-LIUM corpus dataset. To simulate the overlapping speech audios for the two-speaker scenario, we mix two utterances with equal energy on a single acoustic channel using SoX software, one from the target speaker and the other from a background speaker. The same mixture process is applied for each split of the dataset, which we describe as follows. Take the training split for example. Each of the utterances in the split will be used as the target speaker's speech. For each target speech utterance, we randomly select a different speaker's utterance from the same split as the background speech, so that there is no overlap in background speech utterances between different datasets. The length of the resulted mixed audio is as long as that of the target speaker's utterance. Both datasets of the single-speaker and two-speaker scenarios have the same split as the TED-LIUM corpus release 2. 


\subsection{Speaker Embedding Robustness against Environment Variation}

For target speaker embedding extraction, we compare two sources of the target speaker's data as input to the speaker embedding models.

Same Env: Data (a speech segment or face image) from the same environment as the input speech to be recognized is used. It is obtained by randomly selecting another utterance in the same TED talk.

Diff. Env: Data from a different environment as the input speech to be recognized is used. It is obtained by randomly selecting an utterance in a different TED talk given by the target speaker.

The former condition signifies the effect of speaker embedding if we know how the target speaker sounds or looks like in the exact environment as speech recognition. The latter condition represents the situation when we only know what the target speaker sounds or looks like in a different environment, which is a more practical situation and helps us validate the robustness of the target speaker embedding against environment variations.

\subsection{Model Architectures and Training}

Here we describe the architectures of our DNN models. Our acoustic model is a TDNN-based chain model [13]. Let $t$ denote the current timestep. At the input layer, we splice together the 40bin MFCC features of frames at $\{t-1, t, t+1\}$ and the 128dimensional speaker embedding. The first hidden layer is fully connected of 450 nodes. Each of the second to sixth hidden layers has 450 nodes and splices the output of the previous layer at times $\{t-1, t, t+1\},\{t-1,0, t+1, t+2\},\{t-3, t, t+3\},\{t-3, t, t$ $+3\},\{t-6, t-3, t\}$ respectively. The output layer is a softmax function of 3683 phoneme classes. We train with stochastic gradient descent (SGD) with a batch size of 128 frames and an initial learning rate of 0.001 . The input layer of our TDNN-based $\mathrm{x}$-vector model is 40-bin MFCC features. Each of the first 3 hidden layers has 512 nodes and splices the output of the previous layer at times $\{t-2, t-1, t, t+1, t+2\},\{t-2, t, t+2\},\{t-3$, $t, t+3\}$ respectively. The fourth and fifth layers are fully connected of 512 and 1500 nodes. After the statistical pooling layer are two fully-connected layers of 512 nodes. We use SGD with a batch size 64 frames and an initial learning rate of 0.001 . All the nonlinearities for the hidden fully-connected and timedelay layers are rectified linear units followed by batch normalization.

\section{RESULTS AND DISCUSSION}

Table 2 shows the Word Error Rate (WER) of the single-speaker models. Table 3 shows the WER of the two-speaker models. Comparing the results of the single-speaker and two-speaker models, we observe that ASR performance degrades severely in cocktail-party environments compared to single-speaker conditions which have lower background interfering speech. The audio-only baseline without using a static target speaker embedding input for two-speaker cocktail-party problem achieves $65.7 \%$ WER. It is demonstrated that using all three types of speaker embedding as an additional feature can reduce the WER significantly, improving WER to as low as $29.5 \%$. Even in relatively lower noise environments, the results of single-speaker show that using any of the three types of speaker embedding as an additional feature still provides a small gain, reducing the WER from $14.8 \%$ to as low as $14.2 \%$.
Moreover, we compare the performance of the three types of speaker embedding. When the target speaker embedding is extracted using the speaker data from the same environment as speech recognition, i-vector shows better performance than $\mathrm{x}$ vector and f-vector in both single-speaker and cocktail-party conditions. However, when the target speaker embedding is extracted using a different environment's speaker data, $\mathrm{x}$-vector and f-vector outperform i-vector. This suggests i-vector may tend to overfit the exact speaker and environment condition, while $\mathrm{x}$ vector and f-vector show robustness against environment changes. We ascribe this to the fact that i-vector accounts for both speaker and environment acoustic variations simultaneously, while $\mathrm{x}$ vector and f-vector are trained to differentiate speakers, regardless of environments. We also observe x-vector outperforms f-vector in both conditions. While f-vector characterizes the target speaker's identity (or even gender) from the speaker's face image, it does not have acoustic information about the target speaker's voice attributes. On the other hand, $\mathrm{x}$-vector, using speech segment as input for speaker embedding extraction, not only represents the target speaker's identity, but also retains one's voice characteristics.

Table 2. Word Error Rate of single-speaker models

\begin{tabular}{|c|c|c|}
\hline Audio-only & \multicolumn{2}{|c|}{$14.8 \%$} \\
\hline Speaker Embedding & Same Env & Diff. Env \\
\hline i-vector & $\mathbf{1 4 . 2 \%}$ & $15.4 \%$ \\
\hline x-vector & $\mathbf{1 4 . 2 \%}$ & $\mathbf{1 4 . 3 \%}$ \\
\hline f-vector & $14.4 \%$ & $14.4 \%$ \\
\hline
\end{tabular}

Table 3. Word Error Rate of two-speaker models

\begin{tabular}{|c|c|c|}
\hline Audio-only & \multicolumn{2}{|c|}{$65.7 \%$} \\
\hline Speaker Embedding & Same Env & Diff. Env \\
\hline i-vector & $\mathbf{2 9 . 5 \%}$ & $52.4 \%$ \\
\hline x-vector & $34.6 \%$ & $\mathbf{4 1 . 0 \%}$ \\
\hline f-vector & $54.5 \%$ & $51.2 \%$ \\
\hline
\end{tabular}

\section{CONCLUSIONS}

In this work, we present using a static target speaker embedding as additional acoustic model input feature for speaker-targeted speech recognition in cocktail party environments. We investigate three types of text-independent speaker embedding extraction models, using the target speaker's data as simple as a short speech segment or a face image. $i$-vector is extracted by the GMM-UBM with speech segment of the target speaker. $x$-vector is extracted from the bottleneck features of a TDNN speaker recognizer given a speech segment of the target speaker. f-vector is extracted by a CNN of Inception-ResNet architecture trained on triplet loss given an input face image of the target speaker. Empirical evaluation is performed on the TED-LIUM corpus release 2 by overlapping a target speaker and a background speaker's speech signals. We show that using the target speaker embedding reduces WER over the acoustic feature only model from $65.7 \%$ to $29.5 \%$ in twospeaker settings. Comparing the three types of speaker embedding, i-vectors tend to overfit to specific speaker and environment conditions and show the best performance when the environment of speaker data matches that of speech recognition, while $\mathrm{x}$ - 
vectors and f-vectors are more robust with respect to environment variations, as they are trained to differentiate speakers.

\section{REFERENCES}

[1] Alam, M.J., Gupta, V., Kenny, P. and Dumouchel, P. 2015. Speech recognition in reverberant and noisy environments employing multiple feature extractors and i-vector speaker adaptation. EURASIP Journal on Advances in Signal Processing.

[2] Chao, G.-L., Chan, W., and Lane, I. 2016. Speaker-targeted audio-visual models for speech recognition in cocktail-party environments. Interspeech.

[3] Cui, X., Goel, V. and Saon, G. 2017. Embedding-based speaker adaptive training of deep neural networks. Interspeech.

[4] Dehak, N., Kenny, P.J., Dehak, R., Dumouchel, P., and Ouellet. P. 2011. Front-end factor analysis for speaker verification. IEEE Transactions on Audio, Speech, and Language Processing.

[5] Delcroix, M., Zmolikova, K., Kinoshita, K., Ogawa, A. and Nakatani, T. 2018. Single channel target speaker extraction and recognition with speaker beam. IEEE International Conference on Acoustics, Speech and Signal Processing (ICASSP).

[6] Ephrat, A., Mosseri, I., Lang, O., Dekel, T., Wilson, K., Hassidim, A., Freeman, W.T. and Rubinstein, M. 2018. Looking to listen at the cocktail party: a speaker-independent audio-visual model for speech separation. ACM Transactions on Graphics.

[7] Garimella, S., Mandal, A., Strom, N., Hoffmeister, B., Matsoukas, S. and Parthasarathi, S.H.K. 2015. Robust ivector based adaptation of DNN acoustic model for speech recognition. Annual Conference of the International Speech Communication Association.

[8] Guo, Y., Zhang, L., Hu, Y., He, X. and Gao, J. 2016. Msceleb-1m: A dataset and benchmark for large-scale face recognition. European Conference on Computer Vision (ECCV).

[9] Kim, S., Raj, B. and Lane, I. 2016. Environmental noise embeddings for robust speech recognition. arXiv preprint arXiv:1601.02553.

[10] King, B., Chen, I.F., Vaizman, Y., Liu, Y., Maas, R., Parthasarathi, S.H.K. and Hoffmeister, B. 2017. Robust speech recognition via anchor word representations. Interspeech.

[11] Li, C., Ma, X., Jiang, B., Li, X., Zhang, X., Liu, X., Cao, Y., Kannan, A. and Zhu, Z. 2017. Deep speaker: an end-to-end neural speaker embedding system. arXiv preprint arXiv:1705.02304.

[12] Liu, Y. and Kirchhoff, K. 2016. Novel front-end features based on neural graph embeddings for DNN-HMM and LSTM-CTC acoustic modeling. Interspeech.

[13] Povey, D., Peddinti, V., Galvez, D., Ghahremani, P., Manohar, V., Na, X., Wang, Y. and Khudanpur, S. 2016. Purely sequence-trained neural networks for ASR based on lattice-free MMI. Interspeech.

[14] Qian, Y., Chang, X. and Yu, D. 2018. Single-channel multitalker speech recognition with permutation invariant training. Speech Communication.

[15] Rousseau, A., Deléglise, P. and Estève, Y. 2014. Enhancing the TED-LIUM corpus with selected data for language modeling and more TED talks. International Conference on Language Resources and Evaluation (LREC).

[16] Saon, G., Soltau, H., Nahamoo, D. and Picheny, M. 2013. Speaker adaptation of neural network acoustic models using i-vectors. IEEE Workshop on Automatic Speech Recognition and Understanding (ASRU).

[17] Schroff, F., Kalenichenko, D. and Philbin, J. 2015. Facenet: A unified embedding for face recognition and clustering. IEEE Conference on Computer Vision and Pattern Recognition (CVPR).

[18] Snyder, D., Ghahremani, P., Povey, D., Garcia-Romero, D., Carmiel, Y. and Khudanpur, S. 2016. Deep neural networkbased speaker embeddings for end-to-end speaker verification. IEEE Spoken Language Technology Workshop (SLT).

[19] Snyder, D., Garcia-Romero, D., Povey, D. and Khudanpur, S. 2017. Deep neural network embeddings for text-independent speaker verification. Interspeech.

[20] Szegedy, C., Ioffe, S., Vanhoucke, V. and Alemi, A.A. 2017. Inception- $v 4$, inception-resnet and the impact of residual connections on learning. AAAI Conference on Artificial Intelligence.

[21] Tüske, Z., Michel, W., Schlüter, R. and Ney, H. 2017. Parallel neural network features for improved tandem acoustic modeling. Interspeech.

[22] Yu, D., Kolbæk, M., Tan, Z.H. and Jensen, J. 2017. Permutation invariant training of deep models for speakerindependent multi-talker speech separation. IEEE International Conference on Acoustics, Speech and Signal Processing (ICASSP).

[23] Zhao, H., Gan, C., Rouditchenko, A., Vondrick, C., McDermott, J. and Torralba, A. 2018. The sound of pixels. European Conference on Computer Vision (ECCV).

[24] Žmolíková, K., Delcroix, M., Kinoshita, K., Higuchi, T., Ogawa, A. and Nakatani, T. 2017. Learning speaker representation for neural network based multichannel speaker extraction. IEEE Automatic Speech Recognition and Understanding Workshop (ASRU). 